\title{
Constructing complicated carbohydrates
}

A stepwise strategy of targeted synthesis enables researchers to reconstruct cellular glycomes in vitro.

Many of a cell's encounters with the outside world are mediated by a forest of proteinconjugated carbohydrates called $N$-glycans. These molecules comprise diverse sugar groups joined in a myriad of intricately branched structures, and specific $N$-glycan combinations can act as a cell's 'fingerprint'. "It's very common that microbes get into their target cell by hooking onto specific carbohydrates," explains Geert-Jan Boons. "Furthermore, the first interaction between egg and sperm is mediated by carbohydrate-protein interactions."

His team at the University of Georgia is striving to elucidate $N$-glycan function, but the glycome remains poorly charted at present. Isolation of intact $N$-glycans from biological samples is a near-impossible task, and biochemical synthesis typically yields only $\mathrm{N}$-glycans in which every branch contains the same pattern of chemical groups. "This is actually a minority of the glycans found in nature," notes Boons.

Now his group has devised a strategy for precisely controlled synthesis of $\mathrm{N}$-glycans that better mirror the spectrum of glycomic diversity. The team begins with a core pentasaccharide molecule with four potential branch points, each blocked by a different chemical protecting group. Any of these groups can be selectively removed while leaving the other three in place, thereby creating an opening for chemical addition of extra glycan moieties, which can subsequently be extended and modified by hand-picked glycosyltransferase enzymes. This process therefore allows the researchers to design and sequentially construct a host of asymmetrically branched $N$-glycans.

With James Paulson at The Scripps Research Institute in La Jolla, California, Boons designed synthetic glycan microarrays for screening protein-carbohydrate interactions. As a test, they examined variants of the $N$-glycan-binding protein hemagglutinin from influenza virus strains of differing virulence and host preference. Prior studies have identified key trisaccharides that facilitate binding to host cells but have provided little insight into positional effects. "We showed that if you take that trisaccharide and place it in the context of these branched molecules, binding can be gained or lost," says Boons. $\mathrm{He}$ and his colleagues are now endeavoring to clone and express the full complement of human glycan-synthesizing enzymes as a key step toward building a comprehensive panel of 'sugar standards'. "I think we can almost synthesize the full $N$-linked glycome," says Boons. Michael Eisenstein

\section{RESEARCH PAPERS}

Wang, Z. et al. A general strategy for the chemoenzymatic synthesis of asymmetrically branched N-glycans. Science 341, 379-383 (2013). 